\section{PARA A CONSTRUÇÃO DE UMA ESCOLA ATIVA NA DIMENSÃO EXTRACURRICULAR: ESTUDO DE CASO NUMA ESCOLA PORTUGUESA}

\author{
BUILDING AN ACTIVE SCHOOL IN ITS EXTRACURRICULAR \\ DIMENSION: A CASE STUDY IN A PORTUGUESE SCHOOL C
}

\section{PARA LA CONSTRUCCIÓN DE UNA ESCUELA ACTIVA EN LA \\ DIMENSIÓN EXTRACURRICULAR: ESTUDIO DE CASO EN UNA \\ ESCUELA PORTUGUESA CP}

doi) https://doi.org/10.22456/1982-8918.102388

(D) Denise Nogueira Mendes* <denise.n.mendes@hotmail.com>

Fernando Paulo Cunha*** <paulocunha55@gmail.com>

Kelly Lemos O’Hara*** <ohara@ubi.pt>

Paula Maria Batista*<paulabatista@fade.up.pt>

\footnotetext{
*Universidade do Porto. Porto, Portugal.

**Universidade Lusófona. Escola Secundária Almeida Garrett. Porto, Portugal.

***Universidade Beira Interior. Centro de Investigação em Educação e Psicologia da UE. Covilhã, Portugal.
}

\begin{abstract}
Resumo: O propósito central deste estudo é caracterizar uma escola secundária portuguesa (na área do Grande Porto), na sua dimensão extracurricular, como ponto de partida para a implementação de um projeto de Escola Ativa. Utilizamse como instrumentos questionários e entrevistas semiestruturadas, sendo os dados recolhidos tratados com recurso à análise temática e a estatísticas descritivas simples. Os resultados obtidos são apresentados na sequência dos temas encontrados, oportunidades e barreiras, tendo-se concluído que, embora a comunidade educativa mostre interesse na adesão ao projeto, é necessário definir um enquadramento concetual, desenvolver um processo de sensibilização prévia, juntamente com a designação de um líder e uma equipa mobilizadores de uma visão agregadora do mesmo, bem como implementar programas de atividade física motivantes, sistemáticos e sustentáveis.
\end{abstract}

Palavras chave: Ensino fundamental e médio. Atividade motora. Esportes. Professores Escolares.
Recebido em: 28 abr. 2020 Aprovado em: 14 dez. 2020 Publicado em: 18 fev. 2021

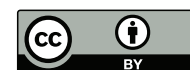

Este é um artigo publicado sob a licença Creative Commons Atribuição 4.0 Internacional (CC BY 4.0). elSSN: $1982-8918$ 


\section{INTRODUÇÃO}

A inatividade física é um aspeto determinante da saúde ao longo da vida, acentuando os riscos de aparecimento de doenças cardiovasculares, cancro do cólon e peito, diabetes, hipertensão, osteoporose, ansiedade e depressão, entre outros (BULL et al., 2020). Estudos recentes relacionados com a mortalidade apontam a inatividade física, em paralelo com o consumo do tabaco e a obesidade, como elementos de risco acrescido para a saúde (DAS; HORTON, 2016).

A Organização Mundial de Saúde, no seu plano global de ação, aponta como alvo, até 2030 , a redução em $15 \%$ da prevalência global de inatividade física em adultos e adolescentes (WORLD HEALTH ORGANIZATION, 2018a).

$\mathrm{Na}$ aceção de BULL et al. (2020), a atividade física (AF) é considerada qualquer movimento produzido pelos músculos esqueléticos requerendo gasto energético, em domínios tão diversificados como o lazer, o trabalho, a educação, em casa e/ou em transporte.

Em crianças e jovens, os benefícios da AF evidenciam uma percentagem mais reduzida de gordura corporal, maior força muscular, marcadores melhorados da saúde cardiovascular e metabólica e maior conteúdo e densidade mineral ósseos (PHYSICAL ACTIVITY GUIDELINES ADVISORY COMMITTEE, 2018; SALLIS et al., 2012). A AF contribui igualmente para melhorar a saúde mental, reduzindo e prevenindo a ansiedade e a depressão, elevando a autoestima e o autoconceito (BROWN et al., 2013; DAS et al., 2016; HOARE et al., 2016; RADOVIC; GORDON; MELVIN, 2017). Parece manifestar-se também uma relação positiva entre a AF, de intensidade moderada-vigorosa, e o funcionamento cerebral (PHYSICAL ACTIVITY GUIDELINES ADVISORY COMMITTEE, 2018; SALLIS et al., 2012; SPRUIT et al., 2016), demonstrando que as crianças mais ativas apresentam maiores recursos atentivos, velocidades superiores de processamento cognitivo e melhores resultados em testes académicos padronizados (DONNELY et al., 2016; ESTEBAN-CORNEJO et al., 2015; GIL-ESPINOSA et al., 2019; LI et al., 2017).

Tradicionalmente, os jovens passam muito tempo na escola, sendo esta um local onde existem preocupações educativas com a saúde e desenvolvimento de projetos e oportunidades neste âmbito que conduzam à aquisição de ferramentas, conhecimentos e comportamentos propiciadores de estilos de vida ativos duradouros (WORLD HEALTH ORGANIZATION, 2018b), reduzindo comportamentos sedentários e incrementando hábitos de AF (VAN DER MARS; LORENZ, 2019).

Na sequência de campanhas de saúde pública destinadas a combater o sedentarismo e os problemas de origem hipocinética, como obesidade, doenças cardiovasculares, diabetes (BIDDLE; GORELY; STENSEL, 2004), excesso de peso e o crescente número de crianças obesas (OGDEN et al., 2016), vários países têm procurado desenvolver o conceito de Escola Ativa (EA), na língua inglesa "Comprehensive School Physical Activity Program" (CORDON; BOURDEAUHUIJ, 2002; MCMULLEN et al., 2015; NAYLOR et al., 2006; REID, 2009, SUTHERLAND et al., 2016). 
A implementação das EA assenta em modelos comportamentais ecológicos (COHEN; SCRIBNER; FARLEY, 2000; HOVELL; WAHLGREN; GEHRMAN, 2002; LOHRMANN, 2010; SALLIS; OWEN; FISHER, 2008), onde o contexto se constitui como determinante poderoso dos níveis de AF (SALLIS et al. 2012). Em consequência, o comportamento relacionado com a saúde pode ser alterado de forma mais eficiente através de envolvimento conjugado a vários níveis: (1) intrapessoal; (2) interpessoal; (3) organizacional/contextual; (4) comunitário e (5) cultural (CARSON et al., 2014; SALLIS et al., 2012; BAUER et al., 2014; SPENCE; LEE, 2003).

O incremento da AF em contexto escolar estrutura-se em seis pontos: (1) assumir uma perspetiva global de escola; (2) considerá-la em todas as decisões políticas; (3) entender a Educação Física (EF) como disciplina nuclear; (4) monitorizar a EF e as oportunidades de AF na escola; (5) assegurar a formação dos professores neste âmbito e (6) garantir a equidade no acesso à AF e à EF (WORLD HEALTH ORGANIZATION, 2018b).

O conceito de EA tem por objetivo nuclear o usufruto diário de 60 minutos de AF moderada-vigorosa, desenvolvendo-se em redor de cinco componentes essenciais: (1) a EF curricular; (2) a AF durante o tempo de escola (integrando o movimento na sala de aula e explorando os recursos proporcionados pelo recreio e o tempo de almoço); (3) AF antes e depois do tempo de escola, a atividade desportiva interna, o desporto escolar e outras potenciais iniciativas, como o transporte ativo; (4) envolvimento do pessoal docente e não docente e (5) envolvimento da família e da comunidade (STELLINO et al., 2019). Face a este quadro, importa esclarecer que a dimensão extracurricular, incorporada neste conceito de EA, engloba a AF na sala de aula (designada como pausa ativa), a AF realizada pelos alunos antes e após os tempos letivos curriculares (por exemplo, no transporte ativo de e para a escola), o desporto escolar (que integra a prática desportiva desenvolvida por grupos-equipas em várias modalidades, com treino regular e competição organizada com outras escolas), a atividade interna (que consiste na realização de diferentes tipos de eventos dentro da própria escola, tais como: o corta-mato, os torneios desportivos interturmas, comemoração do dia Europeu do Desporto, entre outros) e o envolvimento da comunidade escolar, nomeadamente na participação em atividades dirigidas à aquisição/manutenção da aptidão física.

Este estudo tem como propósito central caraterizar uma escola secundária portuguesa (área do Grande Porto) na sua dimensão extracurricular, como ponto de partida para a implementação de uma EA. Especificamente visou-se: (1) captar os entendimentos dos professores coordenadores de departamento e subcoordenadores da área disciplinar em relação à AF na sala de aula; (2) avaliar o interesse dos professores e funcionários na adesão à $A F$ antes, durante ou depois do seu horário de trabalho; (3) examinar a opinião dos alunos relativamente às atividades desportivas realizadas na escola - atividade interna e desporto escolar; (4) avaliar o interesse dos alunos na participação em diversas iniciativas de promoção da AF (exemplo: transporte ativo, salas de aula ativas, programas de caminhadas e corrida); (5) indagar o nível de adesão do diretor, subdiretor da escola, subcoordenador da área disciplinar de EF e coordenador do desporto escolar para a implementação do projeto de EA e (6) apresentar propostas de dinamização da EA, atendendo ao diagnóstico efetuado. 


\section{METODOLOGIA}

\subsection{PARTICIPANTES}

Participaram do estudo cinco coordenadores de departamento (Ciências Experimentais; Ciências Sociais e Humanas; Matemática e Informática; Línguas e Expressões), o coordenador do desporto escolar, três subcoordenadores de departamento (Filosofia; Línguas Germânicas e Ciências Físico-Químicas), o diretor e o subdiretor da escola.

Foram inquiridos 313 alunos, 107 do ensino básico (42 do género feminino, 52 do género masculino e 13 de género não mencionado) e 206 do ensino secundário (136 do género feminino e 70 do género masculino), matriculados nos diferentes cursos da escola, representando $20,9 \%$ do total de alunos da escola $(n=1495)$ (Quadro 1).

Quadro 1 - População de alunos inquiridos

\begin{tabular}{|c|c|c|c|}
\hline Ciclo de Ensino & Gênero & $\begin{array}{c}\text { Frequência } \\
\text { absoluta (FA) }\end{array}$ & $\begin{array}{l}\text { Frequência Relativa } \\
\text { (FR) }\end{array}$ \\
\hline \multirow{3}{*}{ Básico } & 우 & 42 & $13,4 \%$ \\
\hline & $0^{\pi}$ & 52 & $16,6 \%$ \\
\hline & Não mencionado & 13 & $4,1 \%$ \\
\hline \multirow{2}{*}{ Secundário } & 우 & 136 & $43,5 \%$ \\
\hline & $0^{\pi}$ & 70 & $22,4 \%$ \\
\hline
\end{tabular}

Fonte: Inquérito à população escolar

Participaram ainda 65 professores, sendo 56 do género feminino e nove do masculino, representando $56 \%$ do total de professores da escola $(n=116)$. Quanto aos elementos não docentes, participaram 25 assistentes operacionais e assistentes técnicos (100\%), 20 do género feminino e cinco do masculino.

\subsection{INSTRUMENTOS}

$\mathrm{Na}$ recolha de dados, foram utilizados dois instrumentos: a entrevista semiestruturada e questionários de resposta aberta e fechada.

A entrevista semiestruturada permite ao investigador retirar informações e elementos de reflexão muito ricos (BRAUN; CLARKE, 2006; QUIVY; CAMPENHOUDT, 2005), colocando em contacto direto entrevistador e entrevistado, possibilitando verdadeira troca de experiências, perspetivas e "profundidade dos elementos recolhidos"(QUIVY; CAMPENHOUDT, 2005). Aopção pela entrevista semiestruturada justifica-se por dar liberdade ao entrevistado no tratamento dos temas e por se adequar à explicitação de perceções pessoais e experiências, fornecendo respostas ricas e detalhadas (BRAUN; CLARKE, 2013). A realização da entrevista pressupõe a elaboração um guião com questões de natureza aberta, conferindo adaptabilidade ao discurso do entrevistado, quer no próprio questionamento, quer na sequência das questões colocadas (BRAUN; CLARKE, 2006).

Foram realizados dois guiões de entrevista. Um dirigido aos coordenadores e subcoordenadores de departamento, que pretendeu: (1) captar as opiniões dos 
professores relativamente às pausas ativas, (2) avaliar o seu interesse em aderir a momentos de AF nas salas de aula e (3) analisar se os professores se sentem capazes de dinamizar pausas ativas. O outro guião foi dirigido ao diretor e subdiretor da escola, visando: (1) captar a opinião acerca do conceito de salas de aulas ativas e indagar da possibilidade da sua aplicação na escola e (2) perceber a viabilidade da existência de AF para toda a comunidade escolar.

O questionário, enquanto método de recolha de dados, tem como objetivo colocar a um grupo elevado de inquiridos (normalmente representativo de uma população) um conjunto de perguntas relacionadas com a situação profissional, social, as suas opiniões, as suas expetativas, o seu nível de conhecimentos ou outro assunto do interesse do investigador (QUIVY; CAMPENHOUDT, 2005; BURGESS, 2001). Existem dois tipos principais de questões colocadas: (1) de resposta aberta, onde é dada liberdade ao inquirido para responder às perguntas, e (2) de resposta fechada, onde as respostas são estruturadas, escolhendo-se as respostas entre as opções fornecidas (BURGESS, 2001).

A elaboração dos questionários teve em conta quatro características que potenciam o preenchimento por parte dos participantes: (1) interessante, (2) bemapresentado, (3) curto e (4) claramente pensado (JOSSELSON, 2013).

O questionário dirigido aos alunos pretendeu: (1) examinar a sua opinião sobre as atividades desportivas realizadas na escola; e (2) avaliar o interesse na participação em iniciativas de promoção de AF diferentes das habituais. O questionário, dirigido aos professores e assistentes operacionais e técnicos, reportou-se à AF para a comunidade escolar e visava: (1) analisar o nível de prática dos entrevistados; (2) perceber os motivos de ausência de prática; (3) analisar o interesse em participar em atividades desportivas antes, durante e depois do horário de trabalho e (4) examinar quais os tipos de atividades potencialmente mais apelativas para a prática.

\subsection{VALIDAÇÃO DA ENTREVISTA E QUESTIONÁRIO}

A entrevista e o questionário foram elaborados com base na revisão de literatura. A validação do seu conteúdo foi, num primeiro momento, alvo da análise e peritagem de dois doutorados em Ciências do Desporto, especialistas em EF Escolar. Num segundo momento, foi realizada uma entrevista-piloto a um professor de EF pertencente a uma escola secundária da proximidade. Em sequência, foram detetados alguns elementos menos claros e realizados ajustes, na organização e na estrutura do guião, com o intuito de torná-lo mais objetivo e focado na matéria em apreço.

\subsection{PROCEDIMENTOS DE RECOLHA DE DADOS}

As entrevistas foram realizadas individualmente, de forma presencial (algumas com marcação prévia de hora e local), em ambiente calmo e sossegado, durante os segundo e terceiro período do ano letivo 2018/2019, com duração entre dez e 28 minutos, e registadas em sistema áudio digital, num gravador de som portátil. Procurouse que as diferentes questões fossem expostas a todos os entrevistados na mesma 
sequência, contudo, em algumas situações, a sua ordem foi alterada, respeitando o seu fluxo comunicacional. Durante a entrevista, de forma a aprofundar um assunto ou clarificar algumas respostas, foram realizadas perguntas de seguimento.

Para garantir o anonimato e distinguir os vários participantes, foram atribuídas as siglas supramencionadas.

Os questionários colocados aos alunos foram realizados durante as aulas de EF. O segundo questionário foi administrado em duas fases, inicialmente aos professores da escola e, posteriormente, aos funcionários. O questionário aos docentes foi realizado informaticamente. O corpo não docente respondeu ao questionário de forma presencial.

\subsection{TRATAMENTO DE DADOS}

As entrevistas foram escutadas para garantir a familiaridade com o conteúdo registado. Posteriormente, o material foi transcrito em documento Word, o mais fielmente possível ao discurso oral do entrevistado.

O tratamento dos dados foi feito com recurso ao procedimento de análise temática, método qualitativo de análise que permite uma avaliação rica e detalhada dos dados, identificando e referenciando padrões (temas) na matéria recolhida (BRAUN; CLARKE, 2013; BARDIN, 2004).

A análise temática contemplou as seguintes fases: 1) leitura repetida das respostas dos entrevistados, procurando uma familiarização com os dados e permitindo tirar notas sobre assuntos pertinentes; 2) definição dos pontos-chave e características interessantes dos dados; 3) captação das características comuns nos diferentes discursos dos entrevistados e agrupamento das ideias, com definição de temas-padrão e respetiva seleção de excertos representativos; 4) revisão dos diferentes códigos, temas principais e subtemas, descartando alguns devido à falta de pertinência para o objetivo do estudo e 5) atribuição de designação aos diferentes temas e subtemas.

A análise temática resultou na elaboração de dois temas: (1) oportunidades - engloba todos os aspetos favoráveis à implementação do projeto - e (2) barreiras - integra aqueles que limitam a sua operacionalização.

Os dados recolhidos através dos questionários foram tratados com recurso a parâmetros descritivos básicos, especificamente a determinação de frequências absolutas e relativas. No tratamento das respostas às questões abertas foi utilizada a análise semântica, com base nos critérios da relevância e da fácil identificação do conteúdo em relação ao tema equacionado.

\section{RESULTADOS}

\subsection{OPORTUNIDADES}

Os docentes incentivam pontualmente os seus alunos para a prática do exercício físico e demonstram uma atitude positiva acerca da integração do movimento na sala de aula, encontrando vantagens na sua implementação. 
A manifestação de apoio pontual à AF é testemunhada pelo coordenador do departamento de Línguas:

[...] se vem a propósito de alguma coisa que se fala na aula..." e pelo subcoordenador de Ciências Físico-Químicas: “... incentivar os alunos a praticarem AF... claro que isso é um aspeto muito importante para a vida de todas as pessoas, tanto dos alunos como também dos adultos [...].

Quando confrontados com a colocação em prática da AF durante o tempo de aula, alguns docentes expressam uma opinião favorável à sua aplicação, tal como refere o subcoordenador de Línguas Germânicas: "[...] poderia ser para descontrair...". Também poderá ajudar a melhorar o foco mental, como indica o coordenador do departamento de Ciências Sociais e Humanas: “...potencia a concentração...", ou auxiliar na gestão das tarefas e na manutenção da disciplina na aula, opinião manifestada pelo coordenador de departamento de línguas: “...em turmas complicadas... poderia ser uma maneira de eles canalizarem a sua energia... poderia ajudá-los a sossegar e aumentar a concentração...". A mesma ideia é reforçada pelo coordenador do departamento de Ciências Experimentais: “... estes momentos permitem cortar o ritmo, colocar os alunos mais motivados, mais atentos...".

Um dos docentes (coordenador do departamento de Expressões) salienta ganhos potenciais, perante as eventuais desvantagens, na implementação das pausas ativas na sala de aula:

Em relação ao tempo, mas depois compensa, não é? Não podemos escolher o melhor dos dois mundos... Se ajudasse os alunos em termos de concentração, poderia ser uma mais-valia. Em vez de estarmos ali a ralhar com eles e chamá-los à atenção, podia ser mais agradável até do que estar a castigá-los.

A recetividade da administração da escola à ideia é bem patente nas palavras de diretor da escola: "[...] acho que era viável... na perspetiva de criar uma filosofia... criar no projeto educativo algo que fosse central na escola". No entanto, entende que são necessárias a sensibilização e a formação dos docentes para a sua implementação:

[...] identificar exatamente quais são os benefícios, dizer claramente às pessoas que se fizerem isto, acontece aquilo... aproveitar estudos já feitos, que justifiquem e fundamentem esta situação...para as pessoas perceberem exatamente o que estão a fazer, caso contrário fazem-no porque são mandadas... se não valorizam, não resulta.

Os alunos, quando questionados sobre o tema, mostraram maioritariamente $(56,7 \%)$ interesse e curiosidade na utilização de pausas ativas na sala de aula.

No ano letivo 2018/19, 232 alunos $(n=1494)$ participaram no desporto escolar. As modalidades mais praticadas foram o voleibol (58 participantes) e a ginástica (87 participantes), com frequência claramente superior do género feminino $(74,1 \%)$ e com equilíbrio de envolvimento dos níveis de ensino básico e secundário (121 e 111, respetivamente). De notar que o grupo-equipa de voleibol é exclusivamente feminino. Apenas $16,8 \%$ dos alunos inquiridos do ensino básico $(n=107)$ participaram no desporto escolar. No ensino secundário $(n=206)$, existe uma taxa de prática ligeiramente maior $(25,2 \%)$. 
Apesar da percentagem de participantes parecer reduzida, os grupos-equipa estão quase no nível máximo de lotação, como refere o diretor da escola:

Um grupo de desporto escolar não pode ter 200 alunos. Se eu tivesse 200 alunos a quererem desporto escolar, eu não Ihes podia oferecer, porque o professor não tem esse tempo. O grupo de ginástica já não aceita mais ninguém, porque não consegue. O mesmo acontece com o voleibol, esgrima e badminton [...].

$\mathrm{Na}$ atividade interna, os níveis de participação são, no total, de 59,7\% da população inquirida ( $n=313)$, no ensino básico, de 67,3\% ( $n=107)$ e, no secundário, de 55,8\% ( $n=206$ ), com predominância ligeira do género masculino sobre o feminino $(69,6 \%$ e $53,3 \%, n=122$ e 178 , respetivamente). As ações contempladas foram: corta-mato, sessão de treino funcional, "megasprinter" e os torneios internos de voleibol, basquetebol, futebol e andebol, sendo o corta-mato (302 alunos) e o torneio de voleibol (259 alunos) as atividades mais participadas.

A atividade interna parece criar estímulos diferentes nos alunos, como aponta o subcoordenador de EF: "[...] uma experiência e uma vivência diferente... atingimos objetivos que nas aulas não conseguimos [...]".

Os alunos, confrontados com a possibilidade de desenvolver outros tipos de $\mathrm{AF}$, manifestam-se recetivos à sua realização $(76,3 \%)$, salientando-se sobretudo o transporte ativo, caminhar, atividades de ginásio e oferta diferenciada da existente no desporto escolar.

O conceito de transporte ativo merece uma referência particular do coordenador do desporto escolar: "[...] seria uma boa iniciativa, mas, dada a localização da escola, a única possibilidade seria deslocarem-se a pé... assim, viriam pelo passeio... de bicicleta, é difícil, porque não há maneira [...]".

Dos 90 inquiridos (corpo docente e não docente), 49 (54,4\%) afirmam que praticam exercício físico com regularidade, com frequência média de duas vezes por semana. Quem não pratica exercício físico habitualmente $(45,6 \%)$ identifica como principais motivos: falta de tempo e motivação.

A potencial adesão à prática foi manifestada por $68,9 \%$ dos inquiridos, nos níveis 4 a 6 da resposta, sendo que, desses, 32,2\% mostraram "muito interesse" (nível 6) na participação, sobretudo em pilates (32\%) e em caminhadas (32\%), havendo também alguma expressão nas atividades de treino funcional (14\%) e zumba (13\%). Em perspetiva, a maioria $(68,9 \%)$ mostrou interesse em participar em atividades desportivas depois do horário de trabalho.

O subcoordenador de EF e o coordenador do desporto escolar admitem que "[...] os professores e funcionários têm vontade e às vezes pedem para fazer atividades com eles [...]". Na opinião do último, estas atividades fomentariam o espírito de grupo e poderiam gerar mudança comportamental:

Era uma forma de organizar e criar um espírito de grupo... é importante e falta na escola.... Esta ideia é assim reforçada: 'culturalmente nós temos de mudar muito... começar a fazer e depois ver se funciona...', recomendando, por exemplo, uma atividade em particular: 'Criar um grupo de caminhadas na escola... seria a que mais resultava... não me importava de colaborar. [...]. 
O subdiretor da escola salienta a importância de haver quem dinamize as atividades: "Se houvesse aqui algum professor de EF que desse umas aulinhas para professores e funcionários, eu participava". Esta ideia é reforçada pelo diretor: "alguém que coordene a situação e que diga às pessoas o que fazer e como fazer". Refere igualmente a existência de um espaço adequado para a AF:

[...] aquele miniginásio de fitness... foi publicitado para a população escolar, professores, assistentes operacionais, assistentes técnicos, encarregados de educação... teve como ideia principal criar na população educativa outras práticas, não foi só para os alunos... já têm a EF[...].

De acordo com o diretor e o subdiretor, existem oportunidades favoráveis à dinamização do projeto:

[...] a área disciplinar de EF é muito ativa na promoção de atividades e torneios [...] e [...] existem todos os meios e possibilidades disponíveis... o miniginásio à disposição de alunos e professores... espaços exteriores e interiores [...], mas: [... ] é necessário fazer uma sensibilização profunda [...].

\subsection{BARREIRAS}

Os docentes encontram muitas barreiras à colocação em prática da ideia da integração do movimento na sala de aula, invocando sobretudo os seguintes motivos: (i) curta duração da aula; (ii) inovação mal-entendida pelos alunos; (iii) necessidade de cumprimento de programas extensos; (iv) falta de conhecimentos para executar a ideia e (v) desvalorização do projeto.

O constrangimento provocado pela duração da aula é perfeitamente documentado pelo coordenador do departamento de Matemática e Informática:

[...] é um tempo muito curto para os professores cumprirem o que planificaram... Quando tínhamos aulas de 90 minutos, se calhar seria adequado, para haver essa quebra de descontração nos alunos... os 50 minutos são um tempo muito reduzido [...].

Os docentes objetam que a implementação das pausas ativas pode não ser bem entendida pelos alunos, em função da matéria que lecionam, como demonstra o subcoordenador do departamento de Línguas Germânicas: "[...] ser algo de inovador, que nem todos os alunos poderão aceitar, uma vez que sou professora de uma língua estrangeira [...]". Manifestam preocupação na gestão do tempo de aula devido à necessidade de cumprimento dos programas, como assinala o subcoordenador de departamento de Ciências Físico-Químicas: "[...] não temos tempo suficiente para cumprir os programas... muito difícil estar a enquadrar outro tipo de atividades dentro da sala de aula". Não se sentem com competência para levar a cabo a tarefa, sentimento expresso pelo coordenador do departamento Línguas e Expressões: "[...] teria de ter alguma base, alguma preparação [...]" e o subcoordenador do departamento de Ciências Físico-Químicas: "[...], não tenho formação nessa área [...]". Ou, simplesmente, não valorizam o projeto, como afirma o subcoordenador do departamento de Filosofia: "[...] ao não haver razões que justifiquem, não teria razões para aderir a uma medida que não tem urgência [...]" e o coordenador do departamento de Matemática e Informática: "[...] por muitas ferramentas que me dessem, teria sempre bastante relutância em fazer isso dentro da sala de aula [...]". 
No desporto escolar, os principais motivos apontados pelos alunos, explicativos do nível reduzido de prática, são a falta de tempo (76,7\%), incompatibilidade de horário e a oferta de modalidades existente e, de forma residual, o desinteresse, ausência de motivação e a prática desportiva exterior à escola.

Os alunos explicam a não participação na atividade interna devido à falta de tempo e de interesse.

$\mathrm{Na}$ implementação do desporto escolar são evidenciadas dificuldades de origem legal e administrativa, como demonstra o coordenador do departamento de Expressões:

[...] o tempo estipulado para o desporto escolar não é suficiente para se fazer um trabalho de qualidade [...] e o diretor da escola: [...] tem de haver mais professores envolvidos, mais horas disponíveis para as escolas [...], e conclui: [...] um projeto central, que a escola apoie incondicionalmente [...].

No plano das barreiras, o subcoordenador de EF revela alguma apreensão em relação à falta de seriedade e assiduidade dos membros docentes e não docentes: "não conseguiria dar aulas e não levar a situação com regularidade, seriedade e de forma sistemática", mencionando ainda a falta de tempo existente no horário escolar.

Apesar de a escola ter fornecido ferramentas e espaço para a AF dos membros da comunidade educativa, o diretor da escola considera que:

[...] o objetivo não foi atingido', nomeando como principal causa 'o facto de a pessoa que lá esteve, sem incentivo nenhum, não ter conseguido criar um grupo [...]. Aponta ainda limitações organizativas e de enquadramento: $[\ldots]$ haver incentivos para os professores [...].

Como barreira essencial, o diretor aponta que a dinamização da escola deve estar em sintonia com um projeto de âmbito nacional:

[...] deve ser assumido pelo próprio governo na perspetiva de promover uma situação altamente vantajosa para a saúde da população portuguesa... falamos de forma tímida que um país ativo faz a economia da saúde, o problema é que isto não passa de palavras, não é? [...], com forte enquadramento legal e administrativo: criar condições... crédito horário aos professores... para atingir os 60 minutos diários... atividades de sensibilização a nível regional... o governo central e autarquias criando recursos e logística [...].

\section{DISCUSSÃO}

Os resultados evidenciam um conjunto de oportunidades propício à implementação do projeto da EA.

Parece existir uma atitude favorável de alguns docentes na inclusão de pausas ativas nas aulas tradicionais, encontrando alguns potenciais benefícios, sobretudo como elemento de descontração e como quebra do ritmo da lecionação da matéria, melhorando posteriormente a concentração dos alunos.

Estas ideias, embora sumárias, vão de encontro aos dados encontrados na literatura, os quais indicam o valor das pausas ativas, não só como contributo para o acréscimo da AF diária dos alunos, mas também com associações favoráveis 
em alguns indicadores de funcionamento cognitivo (atenção, concentração), comportamentos (conduta em sala de aula) e resultados académicos (classificações nos testes), como apontam os estudos de Mahar (2011), Mahar et al., (2006), Stewart et al., (2004), Tannehill, Van Der Mars e Macphail, (2013), Mears e Jago (2016), Bighle et al., (2019). Segundo Tannehill, Van Der Mrs e Macphail (2013), o desempenho académico pode ser o ponto de "venda" mais interessante para suscitar a recetividade dos professores, caso contrário, podem não estar dispostos a abrir mão do valioso tempo de aula para ajudarem apenas a aumentar a AF dos alunos.

Em contrapartida, como barreiras, os professores manifestam alguma relutância em relação à $\mathrm{AF}$ em sala de aula, apontando o desconhecimento do tema e a falta de formação como aspetos primordiais. Beighle et al., (2019) referem possíveis estratégias para obviar este problema: elaborar skill cards com tarefas, utilizar tecnologia interativa para promover a AF ou simplesmente introduzir experiências que façam mover os alunos dos seus lugares. Os obstáculos ligados à gestão do tempo de aula e aos programas extensos estão relacionados com a pressão provocada pela necessidade de obter bons resultados académicos, numa escola predominantemente do ensino secundário, orientada para prossecução de estudos e sequente ingresso no ensino superior (BEIGHLE et al., 2019).

Outro indicador favorável é a recetividade da comunidade escolar na adesão a programas de AF, sobretudo após o período laboral, aspeto que se interliga com a existência de instalações disponíveis (ginásios, espaço fitness, campo exterior) para os mesmos. Esta intenção enquadra-se naquilo que se designa, no âmbito empresarial, de desenvolvimento de programas geradores de bem-estar no local de trabalho, os quais têm conduzido à obtenção de reais benefícios (PELLETIER, 2011). O trabalho dos professores pode ser muito estressante, levando à exaustão emocional, despersonalização e reduzida realização profissional (VERCAMBRE et al., 2009), para além do desgaste físico inerente, passam longos períodos de tempo em pé (GRIVA; JOAKES, 2003; KITTEL; LEYNEN, 2003; RASKU; KINNUNEN, 2003).

A participação em programas de AF comporta um entendimento multidimensional do bem-estar e assenta num processo autodeterminado, de consciencialização e decisão, direcionado para uma existência mais feliz (LAU et al., 2008). Para além disto, os membros não discentes da comunidade escolar assumem um papel importantíssimo enquanto referências para os estudantes a favor da promoção de uma cultura ligada à AF (TANNEHILL et al., 2013).

Embora exista bastante interesse na participação em programas organizados de AF, constata-se a sua não existência. Beighle et al. (2019) apontam claramente obstáculos a serem vencidos: (1) os custos eventualmente envolvidos; (2) a necessidade de apoio administrativo e diretivo, criando condições facilitadoras sobretudo de ordem laboral; (3) colaboração motivadora da área disciplinar de EF, como dinamizadora do projeto; (4) os participantes têm de ser sujeitos a diagnóstico médico prévio e (5) os programas devem ser individualizados e acompanhados em consonância. Por outro lado, a falta de participação e de abandono está ligada às 
responsabilidades familiares, aos constrangimentos de tempo e à falta de apoio. Ao nível organizacional, a saúde escolar, o clima de escola e a satisfação global com o trabalho de professor moderam a eficácia deste tipo de programas (ERICKSON; GILLESPIE, 2000; JOHNSON et al., 2010).

Importa realçar, como aspeto positivo, os níveis de participação dos alunos no desporto escolar e na atividade interna. Embora o número de envolvidos não seja elevado, esta é uma base da qual se pode partir.

Os programas de AF e desportiva, antes e depois do tempo letivo são uma maneira eficaz de atingir a meta dos 60 minutos diários (WHALEN et al., 2015; BEIGHLE et al., 2019). A literatura aponta numerosos benefícios de saúde, psicológicos e sociais à prática do desporto escolar (EIME et al., 2013; PATE; TROST; LEVIN; DOWDA, 2000), indicando-o ainda como fator preditor da mesma na idade adulta (PERKINS et al., 2004) e após os 70 anos (DOHLE; WANSINK, 2013).

Os resultados do presente estudo vêm ao encontro do que a investigação sugere, isto é, que quer o desporto escolar, quer a atividade interna, apresentam um grande potencial no fomento da AF dos jovens (BOCARRO et al., 2008; EDWARD; KANTERS; BOCARRO, 2014; KANTERS et al., 2008).

A barreira colocada em evidência é o reduzido número e o tipo de modalidades desportivas oferecidas pela escola. Com base nas palavras do subcoordenador de EF e do coordenador do desporto escolar, as escolhas são feitas com base na experiência e interesse dos professores responsáveis, ou seja, sendo sobretudo subordinadas às iniciativas pessoais e muito pouco a uma política estruturada da própria escola. Os professores referem que o tempo destinado ao desporto escolar é muito reduzido (2 horas semanais), pelo que muitos têm de trabalhar gratuitamente, fora do seu horário de trabalho. Como refere o diretor da escola, para potenciar o desporto escolar é essencial um enquadramento legal que crie condições para a sua organização em moldes mais estáveis, sem mudar de professor e/ou modalidade desportiva ano após ano, assumindo-se como um projeto central da escola.

No âmbito da atividade interna, não parece haver uma lógica consistente de atuação, sendo deixada ao livre arbítrio dos professores de EF. A forma esporádica como está organizada (ocorre de três a quatro vezes por período letivo) não parece ter um impacto significativo na consecução do objetivo principal da EA (60 minutos diários de AF), sendo necessário haver um maior grau de sistematicidade na sua realização.

Em geral, foi possível constatar que a escola parece acolher positivamente a ideia da EA, mas o pensamento apresentado parece-nos em geral simplista, genérico e pouco fundamentado. As barreiras apresentadas parecem, a nosso ver, demasiado fáceis de ser vencidas, testemunhando, de algum modo, desconhecimento da dimensão e complexidade do desenvolvimento de um projeto de EA.

Em primeiro lugar, importa que a implementação de uma tarefa dessa natureza assente num enquadramento concetual teórico fundamentador de um processo de pensamento coordenado e sistémico e respetiva elaboração de um plano de operacionalização (STELLINO et al., 2019). Tal reflexão criará porventura as premissas para a integração do conceito no próprio projeto educativo de escola. 
Tal como sugerem Stellino e colaboradores (2019), os constructos das teorias social cognitiva (BANDURA, 1986), do comportamento planeado (FISHBEIN; AJZEN, 1975), de motivação da competência (HARTER, 1978; 1981), da autodeterminação (RYAN; DECl, 2002) e dos sistemas ecológicos (BRONFENBRENNER, 1977; BRONFENBRENNER; MORRIS, 1998) podem funcionar harmoniosamente para delimitar e fundamentar a base teórica da EA, servindo da melhor forma a compreensão, explicação e a desejada promoção da AF.

$\mathrm{Na}$ implementação do projeto, Phelps, Jung e Castelli (2019) apontam como quadro de referência a possibilidade de utilização da estratégia de otimização multifaseada "Multiphase Optimization Strategy" (MOST), que contempla uma primeira fase de preparação, onde se pretende desenvolver a base da implementação e otimização dos multicomponentes, uma segunda, de desenvolvimento do processo e uma terceira (avaliação/reflexão) e quarta (partilha do sucesso) fases, nas quais se procura determinar a relação custo-eficácia dos elementos que atingem o maior número de alunos e fornecer evidências do que foi bem realizado.

Importa salientar que a realização deste estudo se enquadra nesta primeira fase de preparação, ajudando a fazer o diagnóstico da situação, um dos aspetos que ajudará a cumprir os seus objetivos.

No plano interno da própria escola, a realização do projeto passa pela mobilização dos seus recursos humanos, tal como referem Carson et al. (2019), recorrendo a uma tríada capaz de o sustentar e liderar com sucesso: (1) escolher um dinamizador/líder, preferencialmente um professor de EF, (2) a administração da escola, essencial no seu apoio e fomento e (3) uma equipa, aglutinando potenciais elementos influenciadores, professores, pais, que ajudam na coordenação e implementação.

\section{CONCLUSÕES E RECOMENDAÇÕES PARA A IMPLEMENTAÇÃO DA EA}

Os professores mostram interesse, embora pouco fundamentado, na integração do movimento na sala de aula, mas colocam muitas barreiras à aplicação da ideia: falta de tempo para a lecionação da matéria, curta duração da aula, pouca pertinência para a disciplina e ausência de formação para tal.

O corpo docente e não docente revela disponibilidade para participar em programas de AF antes, durante e após o horário laboral.

A participação na atividade interna e no desporto escolar é reduzida, se for tida em consideração a população escolar. Os principais motivos apontados para a pouca adesão são a falta de tempo dos alunos e a oferta considerada pouco atrativa das modalidades desportivas existentes. Paralelamente, não parece existir uma política estruturada e coerente, coordenada com a disciplina de EF, que possibilite atingir maiores índices de participação e sistematicidade.

Os alunos manifestam interesse na participação em iniciativas de promoção da AF, nomeadamente o transporte ativo, possibilidade de integrar o movimento na sala de aula e programa de caminhada. 
Existe, da parte dos órgãos diretivos da escola, boa recetividade à implementação do projeto de EA. Assim, face ao contexto existente, sugerem-se os seguintes aspetos gerais, como suporte para a implementação de um projeto de EA:

(1) Definir o quadro de referência teórico conceptual de base;

(2) Programar uma importante campanha de sensibilização, acentuando potenciais benefícios e fundamentação, mostrando as conclusões de diversos trabalhos de investigação realizados sobre o tema, de modo a construir uma visão entusiasmante sobre ele;

(3) Seguir as indicações mencionadas na literatura: escolher um líder capaz de inspirar/ entusiasmar a comunidade escolar, criar um grupo de trabalho responsável pela dinamização do projeto (eventualmente, aproveitando a equipa já existente do Projeto de Educação para a Saúde) e aproveitar a orientação favorável existente na administração;

No que concerne ao âmbito específico da dimensão extracurricular, apontamse as seguintes potenciais linhas de atuação:

(1) Conduzir um amplo inquérito, abrangendo toda a comunidade escolar, de forma a conhecer detalhadamente as suas necessidades;

(2) Definir uma política coerente relativamente ligando a EF curricular ao desporto escolar e à atividade interna;

(3) Na sequência do ponto anterior, o desporto escolar e a atividade interna devem garantir uma oferta mais diversificada em resposta às opções dos alunos, envolvendo níveis de prática mais sistemáticos, com o intuito de atingir a meta global dos 60 minutos diários de AF moderada-vigorosa;

(4) A igualdade de género na participação deve ser uma preocupação central dos programas a desenvolver;

(5) Oferecer suporte formativo aos docentes das várias áreas disciplinas, para que se sintam mais conscientes e seguros na implementação prática das pausas ativas;

(6) Organizar atividades destinadas a toda a comunidade escolar, nomeadamente no âmbito da aquisição/manutenção da aptidão física, após o horário laboral, garantindo facilidade e sustentabilidade na gestão dos recursos humanos e materiais necessários;

(7) Implementar um programa de transporte ativo, de e para a escola;

(8) Sistematizar a avaliação do projeto, de modo a redefini-lo quando necessário. 


\section{REFERÊNCIAS}

BANDURA, Albert. Social foundations of thought and action. Englewood Cliffs, NJ: Prentice Hall, 1986.

BARDIN, Laurence. Análise de conteúdo. 3.ed. Lisboa: Edições 70, 2004.

BAUER, Ursula; BRISS, Peter; GOODMAN, Richard; BOWMAN, Barbara. Prevention of chronic disease in the 21st century: elimination of the leading preventable causes of premature death and disability in the USA. The Lancet, v. 384, n. 9937, p. 45-52, 2014. DOI: 10.1016/S0140-6736(14)60648-6

BEIGHLE, Aaron; ERWIN, Heather; WEBSTER, Collin; WEBSTER, Michelle. Physical Activity during school. In: CARSON, Russell; WEBSTER, Collin A. (ed.). Comprehensive school physical activity programs: putting evidence-based research into practice. Champaign: Human Kinetics, 2019. p. 99 -109.

BIDDLE, Stuart; GORELY, Trish; STENSEL, David. Health-enhancing physical activity and sedentary behavior in children and adolescents. Journal of Sports Sciences, v. 22, n. 8, p. 679-701, 2004. DOI: https://doi.org/10.1080/02640410410001712412

BOCARRO, Jason; KANTERS, Michael; CASPER, Jonathan; FORRESTER, Scott. School physical education, extracurricular sports, and lifelong active living. Journal of Teaching in Physical Education, v. 27, n. 2, p.155-166, 2008. DOI: 10.1123/jtpe.27.2.155

BRAUN, Virginia; CLARKE, Victoria. Successful Qualitative Research: A Practical Guide for Beginners. Thousand Oaks: Sage, 2013.

BRAUN, Virginia; CLARKE, Victoria. Using thematic analysis in psychology. Qualitative Research in Psychology, v.3, n.2, p. 77-101, 2006. DOI: https://doi. org/10.1191/1478088706qp063oa

BRONFENBRENNER, Urie. Toward an experimental ecology of human development. American Psychologist, v. 32, n. 7, p. 513, 1977. DOI: https://doi. org/10.1037/0003-066X.32.7.513

BRONFENBRENNER, Urie; MORRIS, Pamela. The ecology of developmental processes. In: W. DAMON; R. M. LERNER (Eds.). Handbook of child psychology: Theoretical models of human development. Hoboken: John Wiley, 1998. p. 993-1028.

BROWN, Helen; PEARSON, Natalie; BRAITHWAITE, Rock; BROWN, Wendy; BIDDLE, Stuart. Physical activity interventions and depression in children and adolescents. Sports Medicine, v. 43, n. 3, p. 195-206, 2013. DOI: https://doi.org/10.1007/s40279-012-0015-8

BULL, Fiona; AL-ANSARI, Salih; BIDDLE, Stuart; BORODULIN, Katja; BUMAN, Matthew; CARDON, Greet; WILLUMSEN, Juana. World Health Organization 2020 guidelines on physical activity and sedentary behaviour. British Journal of Sports Medicine, v.54, p. 1451-1462, 2020. DOI: https://doi.org/10.1136/bjsports-2020-102955

BURGESS, Thomas F. A general introduction to the design of questionnaires for survey research. Information systems services: Guide to the Design of Questionnaires. Leeds: University of Leeds, 2001.

CARSON, Russell; ABEL-BEREI, Catherine; RUSS, Laura; SHAWLEY, Jessica; PEAL, Tanya; WEINBERGER, Cyrus. Internal Capacity Building: the role of the CSPAP Champion and other professionals. In: CARSON, R.; WEBSTER, C. A. (Eds.). Comprehensive School 
Physical Activity Programs: Putting Evidence-Based Research into Practice. Champaign: Human Kinetics, 2019. p.35-52.

CARSON, Russell; CASTELLI, Darla; BEIGHLE, Aaron; ERWIN, Heather. School-based physical activity promotion: A conceptual framework for research and practice. Childhood Obesity, v. 10, n. 2, p. 100-106, 2014. DOI: https://doi.org/10.1089/chi.2013.0134

COHEN, Deborah; SCRIBNER, Richard; FARLEY, Thomas. A structural model of health behavior: a pragmatic approach to explain and influence health behaviors at the population level. Preventive Medicine, v. 30, n. 2, p. 146-154, 2000. DOI: https://doi. org/10.1006/pmed.1999.0609

CORDON, Greet; BOURDEAUDHUIJ, Ilse. Physical education and physical activity in elementary schools in Flanders. European Journal of Physical Education, v. 7, n. 1, p. 5-18, 2002. DOI: https://doi.org/10.1080/1740898020070102

DAS, Jai; SALAM, Rehana; LASSI, Zohara; KHAN, Marium; MAHMOOD, Wajeeha; PATEL, Vikram; BHUTTA, Zulfiqar. Interventions for adolescent mental health: an overview. of systematic reviews. Journal of Adolescent Health, v. 59, n. 4, p. S49-S60, 2016. DOI: https://doi.org/10.1016/j.jadohealth.2016.06.020

DAS, Pamela; HORTON, Richard. Physical activity-time to take it seriously and regularly. Lancet, v. 388, n. 10051, p. 1254-1255, 2016. DOI: https://doi.org/10.1016/ $\underline{\text { S01406736(16)31070-4 }}$

DOHLE, Simone; WANSINK, Brian. Fit in 50 years: participation in high school sports best predicts one's physical activity after Age 70. BMC Public Health, v.13, n.1, p.1100, 2013. DOI: https://doi.org/10.1186/1471-2458-13-1100

DONNELLY, Joseph; HILLMAN, Charles; CASTELLI, Darla; ETNIER, Jennifer; LEE, Sarah; TOMPOROWSKI, Philip; LAMBOURNE, Kate, SZABO-REED, Amanda. Physical activity, fitness, cognitive function, and academic achievement in children: a systematic review. Medicine and Science in Sports and Exercise, v. 48, n. 6, p. 1197, 2016. DOI: https://doi.org/10.1249/MSS.0000000000000901

EDWARDS, Michael; KANTERS, Michael; BOCARRO Jason. Policy changes to implement intramural sports in North Carolina Middle Schools: Simulated effects on sports participation rates and physical activity intensity, 2008-2009. Preventing Chronic Disease, v.11, 2014. DOI: https://doi.org/10.5888/pcd11.130195

EIME, Rochelle; YOUNG, Janet; HARVEY, Jack; CHARITY, Melanie; PAYNE, Warren. A systematic review of the psychological and social benefits of participation in sport for children and adolescents: informing development of a conceptual model of health through sport. International Journal of Behavioral Nutrition and Physical Activity, v. 10, n. 1, p. 98, 2013. DOI: https://doi.org/10.1186/1479-5868-10-98

ERICKSON, Jill; GILLESPIE, Catherine. Reasons women discontinued participation in an exercise and wellness program. Physical Educator, v. 57, n. 1, p. 2-7, 2000.

ESTEBAN-CORNEJO, Irene; TEJERO-GONZALEZ, Carlos; SALLIS, James; VEIGA, Oscar. Physical activity and cognition in adolescents: A systematic review. Journal of Science and Medicine in Sport, v. 18, n. 5, p. 534-539, 2015. DOI: https://doi.org/10.1016/j. jsams.2014.07.007

FISHBEIN, Martin; AJZEN, Leek. Belief, Attitude, Intention, and Behavior: An Introduction to Theory and Research. Reading: Addison-Wesley, 1975. 
GIL-ESPINOSA, Francisco; CADENAS-SANCHEZ, Javier; PALMA, Chillón. Physical fitness predicts the academic achievement over one-school year follow-up period in adolescents. Journal of Sports Sciences, v. 37, n. 4, p. 452-457, 2019. DOI: 10.1080/02640414.2018.1505184

GRIVA, Konstadina; JOEKES, Katherine. UK teachers under stress: Can we predict wellness on the basis of characteristics of the teaching job?. Psychology and Health, v. 18, n. 4, p. 457-471, 2003. DOI: $10.1080 / 0887044031000147193$

HARTER, Susan. A model of mastery motivation in children: Individual differences and developmental change. In: COLLINS, W.A. Aspects on the development of competence: The Minnesota Symposia on Child Psychology. Hillsdale: Erlbaum, 1981. v.14, p. 215-255.

HARTER, Susan. Effect and motivation reconsidered. Toward a developmental model. Human development, v. 21, n. 1, p. 34-64, 1978. DOI: $10.1159 / 000271574$

HOARE, Erin; MILTON, Karen; FOSTER, Charlie; ALLENDER, Steven. The associations between sedentary behaviour and mental health among adolescents: a systematic review. International journal of behavioral nutrition and physical activity, v. 13, n. 1, p. 108, 2016. DOI: 10.1186/s12966-016-0432-4

HOVELL, Melbourne; WAHLGREN, Dennis; GEHRMAN Christine A. The behavioral ecological model. In: DiCLEMENTE, Ralph; CROSBY, Richard A.; KEGLER, Michelle. (ed). Emerging theories in health promotion practice and research: Strategies for improving public health. San Francisco: Jossey-Bass, 2002. p. 347-385.

JOHNSON, Carolyn; LAI, Yen-Ling; RICE, Janet; ROSE, Diego; WEBBER, Larry. ACTION live: using process evaluation to describe implementation of a worksite wellness program. Journal of Occupational and Environmental Medicine, v. 52 (Suppl 1), p. S1421, 2010. DOI: 10.1097/JOM.0b013e3181c81ade

JOSSELSON, Ruthellen. Interviewing for qualitative inquiry: A relational approach. New York: Guilford Press, 2013.

KANTERS, Michael; BOCARRO, Jason, CASPER, Jonathan; FORRESTER, Scott. Determinants of sport participation in middle school children and the impact of intramural sports. Recreational Sports Journal, v. 32, n. 2, p. 134-151, 2008. DOI: 10.1123/rsj.32.2.134

KITTEL, France; LEYNEN, Françoise. A study of work stressors and wellness/health outcomes among Belgian school teachers. Psychology and Health, v.18, n.4, p. 501-510, 2003. DOI: $\underline{10.1080 / 0887044031000147229}$

LAU, Patrick; CHAN, Raymond; YUEN, Mantak; MYERS, Jane; LEE, Queenie. Wellness of teachers: A neglected issue in teacher development. In: LEE, John Chi-kin; SHIU, Ling-po (ed.) Developing teachers and developing schools in changing contexts. Hong Kong: Chinese University, 2008. p. 101-116.

LI, Joana; O'CONNOR, Helen; O'DWYER, Nicholas; ORR, Rhonda. The effect of acute and chronic exercise on cognitive function and academic performance in adolescents: $A$ systematic review. Journal of Science and Medicine in Sport, v. 20, n. 9, p. 841-848, 2017. DOI:10.1016/j.jsams.2016.11.025

LOHRMANN, David. A complementary ecological model of the coordinated school health program. Journal of School Health, v. 80, n. 1, p. 1-9, 2010. DOI: $\underline{10.1177 / 003335490812300605}$ 
MAHAR, Matthew. Impact of short bouts of physical activity on attention-to-task in elementary school children. Preventive medicine, V. 52; p. S60-S64, 2011. DOI: https://doi. org/10.1016/j.ypmed.2011.01.026

MAHAR, Matthew; MURPHY, Sheila; ROWE, David; GOLDEN, Jeannie; SHIELDS, Tamlyn; RAEDEKE, Thomas. Effects of a classroom-based program on physical activity and on-task behavior. Medicine and Science in Sports and Exercise, v. 38, p. 2086-2094, 2006. DOI: https://doi.org/10.1249/01.mss.0000235359.16685.a3

MCMULLEN, John; CHROININ, Deirdre, TAMMELIN, Tuij; POGORZELSKA, Malgorzata; VAN DER MARS, Hans. International approaches to whole-of-school physical activity promotion. Quest, v. 67, n. 4, p. 384-399, 2015. DOI: $10.1080 / 00336297.2015 .1082920$

MEARS, Ruth; JAGO, Russell. Effectiveness of after-school interventions at increasing moderate-to-vigorous physical activity levels in 5 to 18 years old: a systematic review and meta-analysis. British Journal of Sports Medicine, v. 50, n. 21, p. 1315-1324, 2016 DOI: 10.1136/bjsports-2015-094976

NAYLOR, Patti-Jean; MACDONALD, Heather; ZEBEDEE, Janelle; REED, Katherine; MCKAY, Heather. Lessons learned from Action Schools! BC —an 'active school' model to promote physical activity in elementary schools. Journal of Science and Medicine in Sport, v. 9, n. 5, p. 413-423, 2006. DOI: 10.1016/j.jsams.2006.06.013

OGDEN, Cynthia; CARROLL, Margaret; LAWMAN, Hannah; FRYAR, Cheryl; KRUSZONMORAN, Deanna; FLEGAL, Katherine. Trends in obesity prevalence among children and adolescents in the United States, 1988-1994 through 2013-2014. JAMA, v. 315, n. 21, p. 2292-2299, 2016. DOI: https://doi.org/10.1001/jama.2016.6361

PATE, Russel; TROST, Stewart; LEVIN, Sarah; DOWDA, Marsha. Sports participation and health-related behaviors among US youth. Archives of Pediatrics \& Adolescent Medicine, v. 154, n. 9, p. 904-911, 2000. DOI: https://doi.org/10.1001/archpedi.154.9.904

PELLETIER, Kenneth. A review and analysis of the clinical and cost-effectiveness studies of comprehensive health promotion and disease management programs at the worksite: update VIII 2008 to 2010. Journal of Occupational and Environmental Medicine, v. 53, n.11, p. 1310-1331.2011. DOI: https://doi.org/10.1097/JOM.0b013e3182337748

PERKINS, Daniel; JACOBS, Janis; BARBER, Bonnie; ECCLES, Jacquelynne. Childhood and adolescent sports participation as predictors of participation in sports and physical fitness activities during young adulthood. Youth \& Society, v. 35, n.4, p. 495-520, 2004. DOI: https://doi.org/10.1177/0044118X03261619

PHELPS, Ashley; JUNG, Yeonhak; CASTELLI, Darla. Multicomponent optimization strategy and CSPAP implementation. In: CARSON, Russell; WEBSTER, Collin A. (ed.). Comprehensive School Physical Activity Programs: Putting Evidence-Based Research into Practice. Champaign: Human Kinetics, 2019. p. 157-169.

PHYSICAL ACTIVITY GUIDELINES ADVISORY COMMITTEE. Physical activity guidelines advisory committee scientific report. Washington, DC: US Department of Health and Human Services, F2-33, 2018.

QUIVY, Raymond; CAMPENHOUDT, Luc Van. Manual de investigação em ciências sociais. Lisboa: Gradiva, 2005.

RADOVIC, Sara; GORDON, Michael; MELVIN, Glenn. Should we recommend exercise to adolescents with depressive symptoms? A meta-analysis. Journal of Pediatrics and Child Health, v. 53, n. 3, p. 214-220, 2017. DOI: https://doi.org/10.1111/jpc.13426 
RASKU, Anne; KINNUNEN, Ulla. Job conditions and wellness among Finnish upper secondary school teachers. Psychology and Health, v.18, no. 4, p. 441-456, 2003. DOI: $\underline{10.1080 / 0887044031000147184}$

REID, Gavin. Delivering sustainable practice? A case study of the Scottish Active Schools program. Sport, Education and Society, v. 14, n. 3, p. 353-370, 2009. DOI: $\underline{10.1080 / 13573320903037879}$

RYAN, Richard; DECI, Edward. Overview of self-determination theory: An organismic dialectical perspective. In: DECl, Edward; RYAN, Richard (ed.). Handbook of selfdetermination research. Rochester: University of Rochester, 2002. p. 3-33.

SALLIS, James; MCKENZIE, Thomas; BEETS, Michael; BEIGHLE, Aaron; ERWIN, Heather; LEE, Sara. Physical education's role in public health: Steps forward and backward over 20 years and HOPE for the future. Research Quarterly for Exercise and Sport, v. 83, n. 2, p. 125-135, 2012. DOI: $10.1080 / 02701367.2012 .10599842$

SALLIS, James; OWEN, Neville; FISHER, Edwin. Ecological models of health behavior. In: GLANZ, Karen; RIMER, Barbara; VISWANATH, K., (ed.). Health behavior and health education. San Francisco: Jossey-Bass, 2008.

SPENCE, John; LEE, Rebecca. Toward a comprehensive model of physical activity. Psychology of Sport and Exercise, v. 4, n. 1, p. 7-24, 2003. DOI: 10.1016/S14690292(02)00014-6

SPRUIT, Anouk; ASSINK, Mark; VAN VUGT, Eveline; VAN DER PUT, Claudia; STAMS, Geert. The effects of physical activity interventions on psychosocial outcomes in adolescents: A meta-analytic review. Clinical Psychology Review, v. 45, p. 56-71, 2016. DOI: $\underline{10.1016 / j . c p r .2016 .03 .006}$

STELLINO, Megan; VAZOU, Spyridoula; KOON, Lyndsie; HODGIN, Katie. Social psychological and motivational theoretical frameworks for CSPAP intervention. In: CARSON, Russell; WEBSTER, Collin A. (eds.). Comprehensive School Physical Activity Programs: Putting Evidence-Based Research into Practice. Champaign: Human Kinetics, 2019. p.67-84.

STEWART, James; DENNISON, David; KOHL, Harold; DOYLE, Andrew. Exercise level and energy expenditure in the TAKE 10! In-class physical activity program. Journal of School Health, v. 74, n. 10, p. 387-400, 2004. DOI: 10.1111/j.1746-1561.2004.tb06605.x

SUTHERLAND, Rachel; CAMPBELL, Elizabeth; LUBANS, David; MORGAN, Philip; NATHAN, Nicole; WOLFENDEN, Luke; OKELY, Anthony; WILLIAMS, Amanda. The physical activity 4 everyone cluster randomized trial: 2-year outcomes of a school physical activity intervention among adolescents. American Journal of Preventive Medicine, v. 51, n. 2, p. 195-205, 2016. DOI: 10.1016/j.amepre.2016.02.020

TANNEHILL, Deborah, VAN DER MARS, Hans; MACPHAIL, Ann. Building effective physical education programs. Burlington: Jones \& Bartlett, 2013.

VAN DER MARS, Hans; LORENZ, Kent. CSPAPs: History, Foundations, Possibilities and Barriers. In: CARSON, Russell; WEBSTER, Collin A. (ed.). Comprehensive School Physical Activity Programs: Putting Evidence-Based Research into Practice. Champaign: Human Kinetics, 2019. p.3-18.

VERCAMBRE, Marie-Noël; BROSSELIN, Pauline; GILBERT, Fabien; NERRIÉRE, Eléna; KOVESS-MASFÉTY, Viviane. Individual and contextual covariates of burnout: a crosssectional nationwide study of French teachers. BMC Public Health, v.9, n. 1, p. 333, 2009. DOI: https://doi.org/10.1186/1471-2458-9-333 
WHALEN, Laurel; MCCAUGHTRY, Nate, GARN, Alex; KULIK, Noel; CENTEIO, Erin; MALJAK, Kimberly; KASETA, Michael; SHEN, Bo; MARTIN, Jeffrey. Why inner-city highschool students attend after-school physical activity clubs. Health Education Journal, v. 75, n. 6, p. 639-651, 2015. DOI: 10.1177/0017896915608885

WORLD HEALTH ORGANIZATION. Global action plan on physical activity 2018-2030: more active people for a healthier world. Switzerland, 2018a.

WORLD HEALTH ORGANIZATION. Promoting Physical Activity in the Education Sector. Switzerland, 2018b. 
Abstract: The main purpose of this study is to characterize a Portuguese secondary school in the Greater Oporto area, in its extracurricular dimension, as a starting point for implementing a project for an Active School. Questionnaires and semistructured interviews are used as instruments and the data collected are treated with thematic analysis and simple descriptive statistics. After themes, opportunities and barriers are described, the results obtained are presented. The work concludes that, although the educational community shows interest in joining the project, a conceptual framework has to be defined and a previous awareness raising process has to be developed. Furthermore, a leader and a team have to be appointed to mobilize an aggregating vision of that process as well as to implement motivating, systematic and sustainable physical activity programs.

Keywords: Education, primary and secondary. Motor activity. Sports. School Teachers

Resumen: El objetivo principal de este estudio es caracterizar una escuela secundaria portuguesa (en el área del Gran Oporto), en su dimensión extracurricular, como punto de partida para la implementación de un proyecto de Escuela Activa. Se utilizan como instrumentos cuestionarios y entrevistas semiestructuradas, y los datos recopilados se tratan mediante análisis temática y estadísticas descriptivas simples. Los resultados obtenidos se presentan a continuación de los temas encontrados, oportunidades y barreras, y se ha concluido que, aunque la comunidad educativa muestra interés en unirse al proyecto, es necesario definir un marco conceptual, desarrollar un proceso de sensibilización previa, junto con la designación de un líder y un equipo que movilicen una visión integradora del mismo e implementen programas de actividad física que sean motivadores, sistemáticos y sostenibles.

Palabras clave: Educación primaria y secundaria. Actividad motora. Deportes. Profesores. 


\section{LICENÇA DE USO}

Este é um artigo publicado em acesso aberto (Open Access) sob a licença Creative Commons Atribuição 4.0 Internacional (CC BY 4.0), que permite uso, distribuição e reprodução em qualquer meio, desde que o trabalho original seja corretamente citado. Mais informações em: http://creativecommons.org/licenses/by/4.0

\section{CONFLITO DE INTERESSES}

Os autores declararam que não há conflito de interesses neste trabalho.

\section{CONTRIBUIÇÕES AUTORAIS}

Denise Mendes: Desenvolvimento e operacionalização do projeto

Fernando Cunha: Concepção e desenvolvimento do projeto

Kelly O'Hara: Consultoria e cooperação no projeto

Paula Batista: Concepção e desenvolvimento do projeto

\section{FINANCIAMENTO}

O presente trabalho foi realizado com apoio do Centro de Desenvolvimento de Pesquisa em Políticas de Esporte e de Lazer da Rede Cedes do Estado de Mato Grosso - Ministério da Cidadania, Secretaria Especial do Esporte/Secretaria Nacional de Esporte, Educação, Lazer e Inclusão Social (SNELIS); Centro In

\section{ÉTICA EM PESQUISA}

Este estudo foi aprovado pela Comissão de Ética da Faculdade de Desporto da Universidade do Porto.

\section{COMO REFERENCIAR}

MENDES, Denise Nogueira; CUNHA, Fernando Paulo; O'HARA, Kelly Lemos; BATISTA, Paula Maria. Representações da comunidade escolar sobre o conceito de escola ativa: estudo de caso em escola secundária. Movimento (Porto Alegre), v. 27, e27013, jan./dez. 2021. Disponível em: https://seer.ufrgs.br/ Movimento/article/view/102388. Acesso em: [dia] [mês abreviado]. [ano]. DOI: https://doi.org/10.22456/1982-8918.102388

\section{RESPONSABILIBADE EDITORIAL}

Alex Branco Fraga*, Elisandro Schultz Wittizorecki*, Ivone Job*, Mauro Myskiw*, Raquel da Silveira*

*Universidade Federal do Rio Grande do Sul, Escola de Educação Física, Fisioterapia e Dança, Porto Alegre, RS, Brasil 